\title{
Adaptive aerodynamic part feeding enabled by genetic algorithm
}

\author{
Sebastian Blankemeyer ${ }^{1} \cdot$ Torge Kolditz $^{1} \cdot$ Jan Busch $^{2} \cdot$ Melissa Seitz $^{2} \cdot$ Peter Nyhuis $^{2} \cdot$ Annika Raatz $^{1}$
}

Received: 7 May 2021 / Accepted: 24 August 2021 / Published online: 18 September 2021

(c) The Author(s) 2021

\begin{abstract}
Aerodynamic feeding systems represent one possibility to meet the challenges of part feeding for automated production in terms of feeding performance and flexibility. The aerodynamic feeding system investigated in this article is already able to adapt itself to different workpieces using a genetic algorithm. However, due to the operating principle, the system is susceptible to changes in environmental conditions such as air pressure and pollution (e.g. dust). To minimise the effect of ambient influences, the system must be enabled to detect changes in the feeding rate and react autonomously by adapting the system's adjustment parameters. In this work, based on pre-identified factors interfering with the aerodynamic orientation process, a new approach is developed to react to changes of the ambient conditions during operation. The presented approach makes us of an alternating sequence of monitoring and corrective algorithms. The monitoring algorithm measures the ratio of correctly oriented parts to the total number of fed parts of the process and triggers the corrective algorithm if necessary. Simulated and experimental results both show that an increased feeding rate can be achieved in varying conditions. Furthermore, it is shown that integrating both known process and parameter information can reduce the time for re-parametrisation of the feeding system.
\end{abstract}

Keywords Feeding technology $\cdot$ Flexible manufacturing system $\cdot$ Optimisation $\cdot$ Genetic algorithm

\section{Introduction}

Modern production is characterised by shorter product life cycles, increasing numbers of variants and high cost pressure $[1,12]$. As a result, production systems must offer adaptability options for changes to ensure flexibility [11]. Being part of the assembly stage, feeding systems are responsible for up to $75 \%$ of the costs for automated assembly systems [8]. Hence, one of the main challenges in the field of assembly is the flexibility of feeding technology $[6,14]$, for example by expanding the product portfolio of a system. The most commonly used feeding systems are vibratory bowl feeders $[2,15]$. While being simple and reliable with regard to control and electronic components, vibratory bowl feeders are often designed to feed one particular workpiece, causing a

Sebastian Blankemeyer

blankemeyer@match.uni-hannover.de

1 Institute of Assembly Technology, Leibniz Universität Hannover, An der Universität 2, Garbsen 30823, Germany

2 Institute of Production Systems and Logistics, Leibniz Universität Hannover, An der Universität 2, Garbsen 30823, Germany lack of flexibility [14]. Consequently, a lot of research aims at increasing the flexibility of commonly used systems by integrating adjustable $[10,17]$ or completely interchangeable mechanical orienting devices [15]. The Institute of Production Systems and Logistics (IFA) at Leibniz University Hannover took a different approach and developed an aerodynamic part feeding system that has proven itself as a good alternative to conventional feeding technologies $[9,16]$. A genetic algorithm developed in previous research activities enables the part feeding system to adapt to new workpieces independently [5]. However, changing ambient conditions during operation could reduce the performance and make a re-adjustment of the system necessary. In this article, a method to increase the performance level of the feeding system is developed. Based on an adapted genetic algorithm, the feeding system is enabled to react to the changes in an efficient way. This method is validated using simulation and test series on the aerodynamic feeding system. 


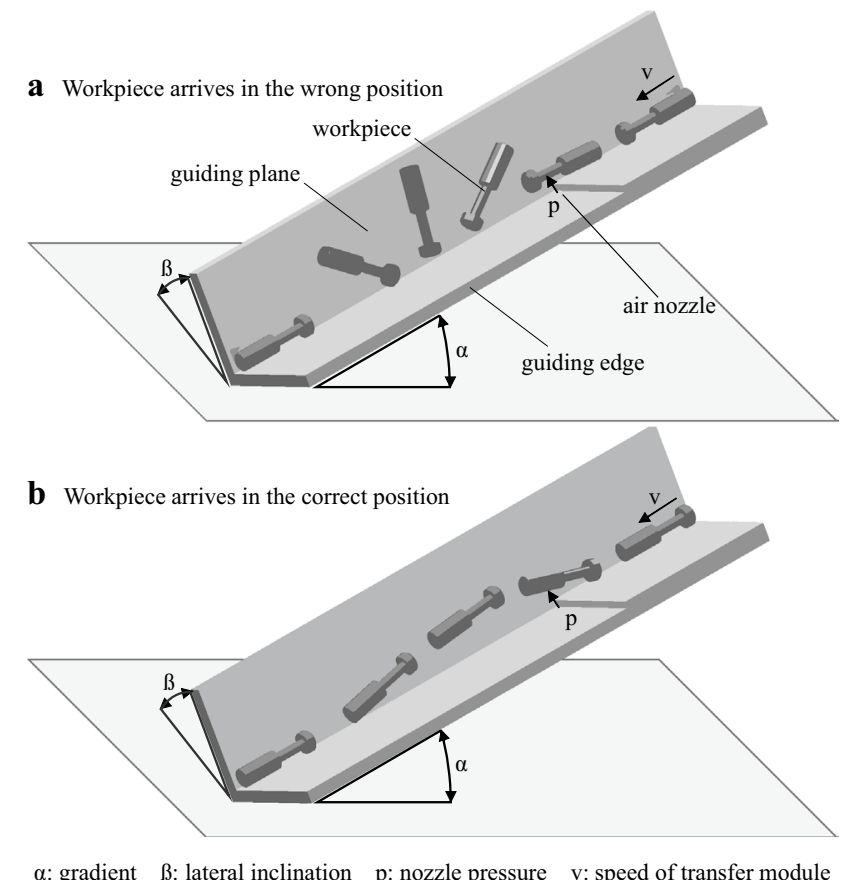

Fig. 1 Aerodynamic orientation process (following [3])

\section{Basics}

The aerodynamic part feeding system developed at the IFA is characterised by a high process speed of up to 800 parts per min [5]. In this part feeding system, workpieces are first separated by a centrifugal feeder and are then oriented aerodynamically. As the orientation of workpieces to be fed is essential in mass production systems [7], the article focuses on the according process. In aerodynamic part feeding, the workpieces are actively transferred from the original orientation of the workpieces to a defined final orientation through a homogeneous air stream. The open design, without mechanical orienting devices, prevents the workpieces from getting stuck, enabling a highly reliable process [5]. The aerodynamic orientation process is based on the asymmetry of the workpieces, which can result from an eccentric centre of gravity, the projected form or local differences in the aerodynamic drag. The orientation takes place on an inclined plane, which consists of two planes that are arranged at a right angle, building a guiding plane and a guiding edge (see Fig. 1). First, a centrifugal feeder separates the parts. A transfer module conveys the separated workpieces to the inclined plane at a speed of v. The gradient angle $\alpha$ causes the parts to slide down the inclined plane and the lateral inclination $\beta$ ensures that the workpieces are aligned with the guiding edge before and after the orientation process. On the slide, the parts pass an air stream, produced by an air nozzle at a constant nozzle pressure $\mathrm{p}$, which initiates the orientation process.
The selected adjustment parameters of the aerodynamic part feeding system ensure that the generated drag forces cause misaligned workpieces to rotate while permitting correctly oriented workpieces to pass through without manipulation [3].

A variant-flexible application of the aerodynamic part feeding system can be realised by adapting the adjustment parameters. However, manual configuration is timeintensive and calls for pronounced expert knowledge [5]. To reduce the setting time and waive the need for qualified personnel, the feeding system was equipped with an algorithm enabling the system to find a suitable parameter configuration autonomously. Since the solution space is non-linear and continuous differentiability cannot be guaranteed, conventional, gradient methods could not be used for this optimisation problem. Therefore, an evolutionary algorithm, specifically a genetic algorithm, was chosen. In the genetic algorithm, possible solutions in the form of individual configurations of the parameters $\alpha, \beta$, $\mathrm{p}$ and $\mathrm{v}$ (chromosomes) are referred to as individuals (see Fig. 2). The orientation rate refers to the proportion of correctly oriented workpieces in the overall quantity of fed workpieces. A chromosome's fitness therefore equals the orientation rate. A criterion, e.g. a maximum number of iterations and a desired feed rate, are set for completion of the algorithm (following [13]). For a detailed description of the system configuration, see Busch et al. [5].

The optimisation described above is only reliable in constant environmental conditions. Consequently, changes in conditions may reduce system performance during operation. The following section describes the development of a method that enables dynamic adaptation of the adjustment parameters and the respective validation to overcome the challenge of unstable environmental conditions.

\section{Development of a method for responding to changing ambient conditions}

Many factors influence the orientation process and thus the orientation rate. An overview of the currently known parameters can be seen in Fig. 3.

Three types of factors can be identified: machine, material and milieu. The machine parameters can be adjusted specifically to the process, as mentioned above. These parameters are feedback controlled, so the values are measured continuously. In contrast, there are parameters that are not measured or cannot be specifically adjusted. The component parameters are generally known and can often be obtained from CAD data. Nevertheless, small deviations can occur across batches due to production uncertainties, which can have a minor influence on the result. Changes in the environment pose the greatest challenge for the feeding process 
Fig. 2 Genetic algorithm for identification of optimal parameter configurations in the aerodynamic part feeding system (following [5])

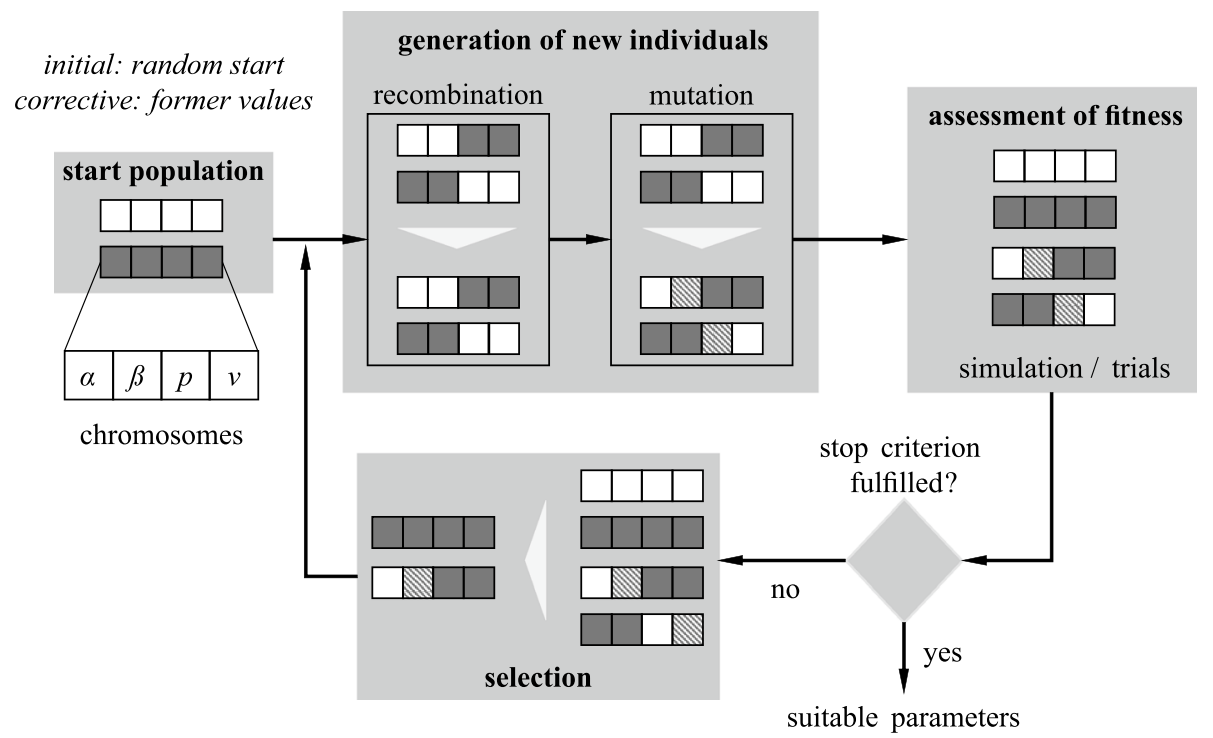

$\alpha$ : gradient

B: lateral inclination

p: nozzle pressure

v: speed of transfer module because it is not possible to make specific adjustments. Since parameters such as the ambient air pressure or changes in the coefficient of friction of the inclined plane have an influence on the forces applied to the workpiece, the feeding rate is affected. Changes in ambient pressure and air density also have an influence on the velocity of the air jet and the air resistance acting on the moving workpiece, which impacts the overall orientation process. The wear of guiding elements of the system and contamination of the inclined plane by dust or other substances affect the speed of the workpiece in the feeding process. This has an effect, for example, on the rotation of the workpiece when passing the nozzle. Due to the resulting changes in the orientation rate, the parameters initially determined by the genetic algorithm may therefore no longer result in a good feeding performance. To define effects of the aforementioned factors on the orientation process, a first analysis was carried out in [3], based on a validated simulation already presented in [4]. For the analysis, a simulation was used to examine the impact of air pressure and friction, because preliminary considerations have shown that these have a large share in influencing the feeding rate. The results, as seen in Fig. 4, show that the coefficient of sliding friction has a great impact on the orientation rate. The value fluctuates between approx. $100 \%$ at a sliding friction coefficient of $\mu \approx 0.17$ and less than $40 \%$ at $\mu \approx 0.23$ (see Fig. 4a). The impact of ambient pressure is relatively small in comparison to the coefficient of friction. The orientation rate was examined between the limits of $936 \mathrm{hPa}$ and $1024 \mathrm{hPa}$ and varies only within a range of 3\% (see Fig. $4 \mathrm{~b}$ ). The results show that even small changes can have a great impact on the output of the feeding system, especially for the coefficient of friction. To increase the efficiency and robustness of the system, a method needs to be developed that continuously monitors the state of the system and reacts quickly to changed situations.

\subsection{Development of a fast adjustment method}

One option for enabling the aerodynamic part feeding system to adapt automatically would involve monitoring all influencing factors with sensors. If a deviation is registered that exceeds a previously defined limit, an automatic preventive re-parameterisation is initiated and the currently measured values are stored as new reference data. It must be considered that only known and easily measurable influence parameters like temperature, air pressure or humidity can be monitored with this approach. Further influencing factors that are hard to determine, such as wear and soiling, are not quantifiable during operation. Furthermore, the interrelations of the ambient parameters with the feeding performance must be known beforehand, so that there are no unnecessary adjustments, reducing the performance.

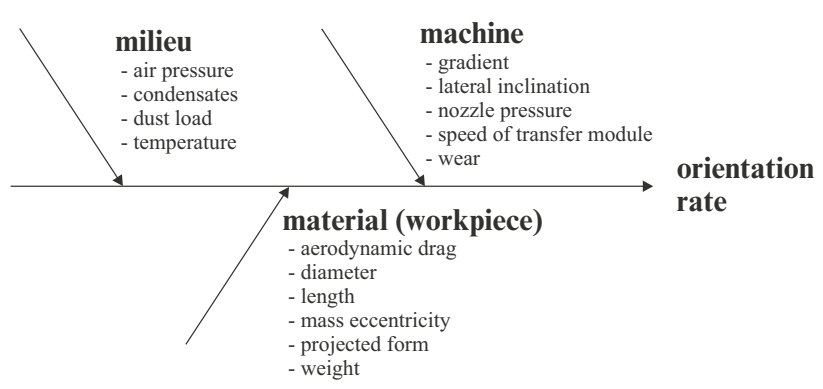

Fig. 3 Influence factors on the orientation rate of the feeding system (following [3]) 

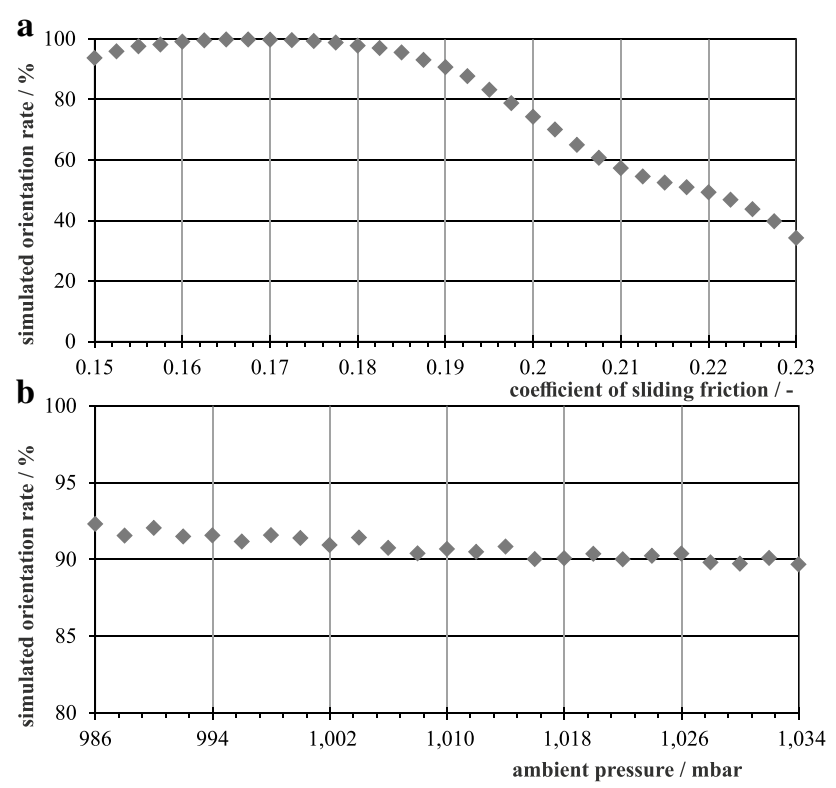

Fig. 4 Orientation rates if the sliding friction coefficient and ambient pressure are varied (following [3])

Alternatively, triggering the reactive re-parameterisation of the part feeding system via the feeding result (in this case the current orientation rate), gives the opportunity to react to hardly quantifiable factors. For this purpose, the current orientation rate is checked in fixed intervals, to monitor if it falls below a previously defined limit during operation. This triggers the automatic re-parameterisation to bring the feeding system back to an optimal state. Thus, interfering factors that are hard to measure are attended to by the system's adaptation to unforeseen changes in ambient conditions. In addition, this automatic adaptation enables the system to react to disturbances that were not previously considered. Consequently, this approach was chosen for implementation on the aerodynamic feeding system. The method for adapting the aerodynamic part feeding system to these changing conditions is shown in Fig. 5. It consists of three parts. First, the initial parameters of the feeding system are determined using the genetic algorithm. In the second part, inspection intervals are used to monitor the current feeding rate and thus the state of the system. The current orientation rate $\mathrm{O}_{\text {cur }}$ is continuously registered in predefined inspection intervals. Subsequently, it verified that $\mathrm{O}_{\text {cur }}$ is above a defined limit $\mathrm{O}_{\mathrm{lim}}$. If the value does not fall below the limit, the next monitor interval follows. In the event that changes in the ambient conditions lead to the limit value being exceeded, the corrective algorithm is initiated.

The basis for the corrective algorithm is the initial algorithm presented in Fig. 2. As soon as the inspection interval has detected a deviation, the algorithm starts to generate the initial population. In contrast to the initial genetic algorithm, this is no longer random, but is assigned the current setting parameters of the plant as well as the second solution found during the last configuration. Since the workpieces and their respective properties remain unchanged, this is expected to reduce the setting time of the algorithm compared to a random initial population. In addition, significant changes in environmental conditions do not usually occur suddenly or dramatically, so it is expected that new solutions will be found more quickly using the previously optimal ones. This assumption is made by assuming that small changes in conditions result only in small variations in the controllable variables. Similar to the initial algorithm, the population size (two parents and two children per generation), mutation rate (55\%), and recombination method (uniform crossover) are specified. For the selection method, a combination of elite and roulette wheel selection was chosen. The best individual of a generation is selected via elite selection and one of the remaining three individuals of the generation is selected via roulette wheel selection. After the optimum parameter combination for the new environmental conditions has been found, a further monitoring interval $i+1$ follows.
Fig. 5 Sequence of the process of the aerodynamic part feeding system's dynamic adjustment to varying sliding friction coefficients and ambient pressures (following [3])

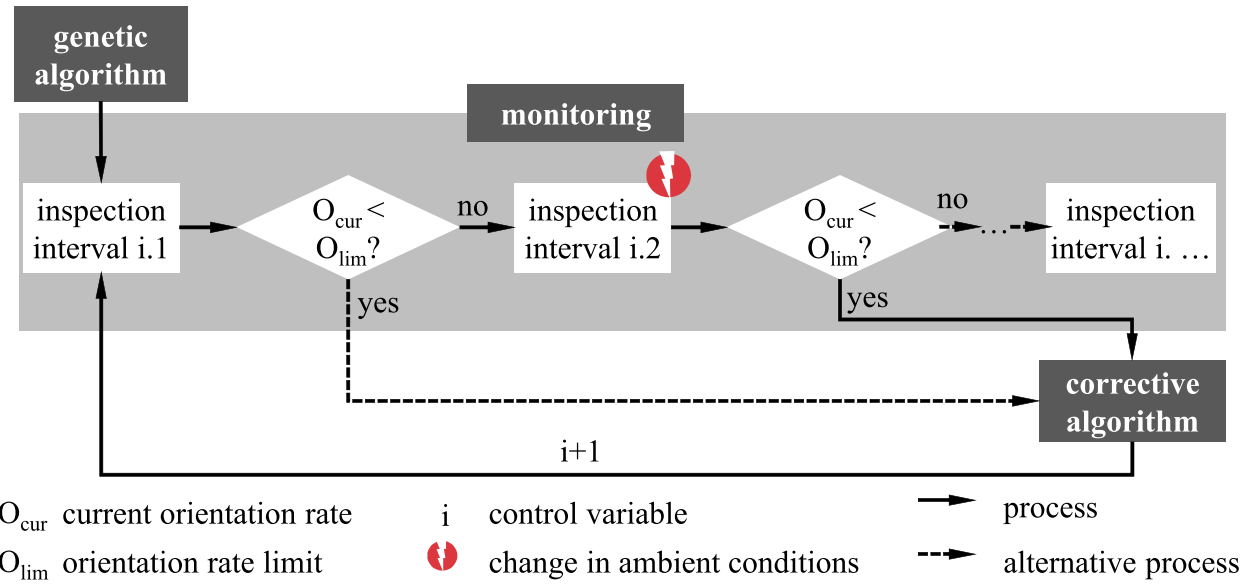




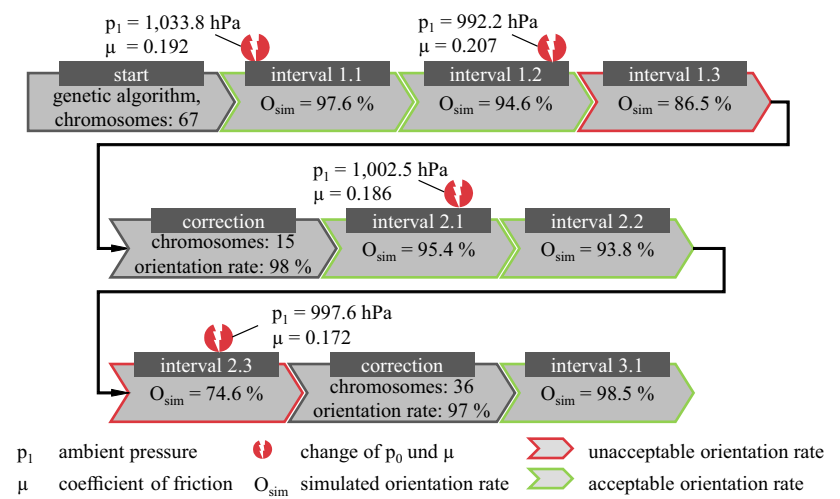

Fig. 6 Simulation results of the adaptive adjustment of the aerodynamic part feeding system to varying sliding friction coefficients and ambient pressures

\subsection{Evaluation in the simulation environment}

In the first step, the functionality of the developed adjustment method using the corrective algorithm is evaluated using the simulation model of the aerodynamic feeding system [4]. For the simulated test runs, the inspection interval is set to 400 workpieces. The desired orientation rate is set to $97 \%$ and the limit of the orientation rate below which a re-parameterisation is necessary $\mathrm{O}_{\lim }$ is exemplary set to $90 \%$ for this investigation. Previous research has shown that using $97 \%$ as desired orientation rate can reduce the number of individuals needed by $40 \%$ compared to the use of $99 \%$ [3]. The changes in the sliding friction coefficient and ambient pressure are applied at a very high frequency that would not occur in practice, to enable observation of the desired effects without needing an unnecessarily long simulation. One exemplary result of the simulation is shown in Fig. 6. After the first run of the genetic algorithm and the corresponding identification of a parameter combination leading to an orientation rate of $97 \%$ or above, the sliding friction coefficient and ambient pressure change within test interval 1.1. This change does not bring about a re-parameterisation of the part feeding system because the orientation rate remains above the required orientation rate $\mathrm{O}_{\mathrm{lim}}$ of $90 \%$. The following change in the ambient conditions in interval 1.2 leads to a reduction in the orientation rate $\mathrm{O}_{\text {sim }}$ from 94.6 to $86.5 \%$ in interval 1.3 . The corrective algorithm is triggered as a consequence. It only requires 15 chromosomes to identify a new parameter configuration that leads to an orientation rate of $97 \%$ or above. In the following interval 2.1 , there is another change in the sliding friction coefficient and ambient pressure, which does not necessitate the corrective algorithm to be triggered. The fourth change in interval 2.3 makes a further re-parameterisation necessary. This requires 36 chromosomes to reach the desired orientation rate of $97 \%$ or more.

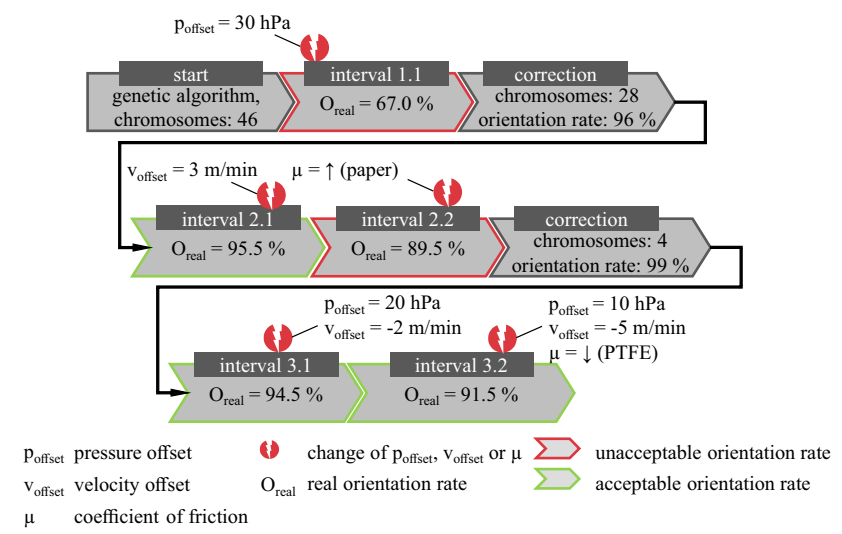

Fig. 7 Exemplary result of the adaptive adjustment of the aerodynamic part feeding system to varying pressure, conveyor velocity and coefficient of friction

Looking at the simulation results, it can be said that the adapted genetic algorithm is working as intended and is suitable to react to fluctuating ambient conditions. A high orientation rate can thus also be realised in varying ambient conditions. Furthermore, it was observed that the correction of the parameter configuration converged faster in the performed simulation than in the initial identification of possible parameter values. With the randomly generated starting population, 67 chromosomes were required, whereas only 15 and 36 chromosomes were needed in the corrective algorithm run in the case being considered.

\subsection{Evaluation on the aerodynamic feeding system}

Having validated the adjustment method in the simulation environment, the corrective algorithm is experimentally evaluated on the real feeding system. To do so, the algorithm is implemented in the control of the aerodynamic feeding system. With this adaption, a test series is conducted to prove the developed approach. For the evaluation, the inspection interval is set to 200 workpieces to reduce the test effort. The desired orientation rate is set to $95 \%$ and the limit of the orientation rate, below which a re-parameterisation is necessary, is set to $90 \%$. Analogous to the simulation, an option must be integrated to manipulate changes in the disturbance at the beginning of the test intervals. Therefore, variable offsets of the parameters $p$ and $v$ were implemented in the machine control to influence the velocity of the air jet when leaving the nozzle and the sliding speed of the workpieces. Additionally, the coefficient of friction on the guiding plane can be altered by applying paper with a higher coefficient of friction than the PTFE-film usually used. The changes are applied at a very high frequency and with great impact to observe the desired effects within a reasonable time. One exemplary result of the evaluation is shown in 
Fig. 7, analogous to Fig. 6. The initial configuration of the genetic algorithm needs 46 chromosomes to identify a suitable parameter combination leading to an orientation rate of $95 \%$ or above. After determining the initial parameters, the air pressure changes within test interval 1.1. Thus, a re-parameterisation of the part feeding system is triggered because the orientation rate $\mathrm{O}_{\text {cur }}$ of $67 \%$ drops below the required orientation rate $\mathrm{O}_{\mathrm{lim}}$ of $90 \%$. Consequently, the corrective algorithm is triggered. To identify a new parameter configuration only 28 chromosomes are required. Compared to the randomly generated starting population of 46 chromosomes, this is a reduction of approx. $40 \%$. The increase of the transfer module speed in interval 2.1 does not reduce the orientation rate below $90 \%$. In the following interval 2.2, there is a change in the sliding friction coefficient, which triggers the corrective algorithm. This time, only four chromosomes are required to reach an orientation rate above $95 \%$. The changes in intervals 3.1 and 3.2 do not lead to a re-parameterisation.

Looking at the results, it can be concluded that a high orientation rate can also be realised in varying ambient conditions on the real feeding system. The re-parameterisation of the process is triggered whenever the orientation rates fall below the limit set beforehand. In addition, the results show that the correction of the parameter configuration converged more quickly than in the initial identification of possible parameter values where the starting population is defined randomly. In each of the 10 test runs, the corrective algorithm was triggered two to three times, depending on the system's reaction to changes in conditions. Of the 27 times the corrective algorithm was triggered, 19 executions were faster than the respective run of the starting algorithm. On average, the corrective algorithm required 30\% fewer chromosomes than the starting algorithm. The duration of the reconfiguration depends on the centrifugal feeder's performance and the number of workpieces used to assess the chromosome's fitness. Assuming that 100 workpieces are enough to do so and given the feeding input of up to 1000 workpieces per min, the resulting duration for re-adjustment ranges between 0.4 and $6.4 \mathrm{~min}$. It can be concluded that the use of a corrective algorithm often leads to a reduction of the needed chromosomes and thus to a decreased system downtime. Furthermore, it can be observed that changing multiple factors simultaneously does not necessarily have a higher impact on the orientation rate than just changing one factor. This indicates that there are still unknown interrelations between the factors, partially compensating each other. This confirms the assumed disadvantage of the initially proposed adjustment method-using sensors to monitor the ambient parameters - that the interrelations would have to be known a priori to determine corresponding value ranges or even dynamically adapt to them (see Sect. 3.1). The approach developed in this article circumvents this problem, since the changes are triggered based on the actual feeding performance and are therefore independent of the type of impact factors and their interrelations.

\section{Discussion}

Simulative and experimental investigations show that the aerodynamic feeding system can uphold a high feeding performance, even in changing ambient conditions. Comparison between the results obtained via simulation and in experiments on the real feeding system shows good congruence. Nevertheless, while the parameters can be controlled in the simulation, it cannot be ruled out that additional, unknown parameters influence the actual feeding process. The investigation indicates that the reconfiguration of the system parameters can be accelerated by reusing previous configurations as starting population for the corrective algorithm. Although ten test runs on the real feeding system have shown this clear tendency, long-term tests are necessary to confirm the results. However, no adverse developments are expected, since the correction algorithm always starts with the previously best-rated configurations. If the ambient conditions change very strongly and suddenly, an algorithm with a random starting population might find a suitable configuration faster, by chance. While the corrective algorithm itself may be limited to the specific use-case of the aerodynamic feeding system, the underlying method can be implemented into existing production environments. Often, the reliability of a process and the detection of disturbances depend on the ability of workers or complex models to adjust the process accordingly. Using this method, performance fluctuations can be identified earlier and countermeasures can be initiated before the entire production system is disrupted. The black-box approach of the method presented in this article simplifies an automatic adjustment approach because the process and the environment do not have to be modelled for each new workpiece. Instead, only the output of the process is considered. Regarding a production environment, this leads to a reduction of expensive downtime and, as a result, a reduced impact on downstream processes. At the same time, the black-box approach reduces the explainability of performance fluctuations, since no process parameters are considered. Therefore, a minor, undetected disturbance might lead to frequent unnecessary reconfigurations in a fully automated environment. Further research may be conducted with regard to the parameters of the developed adjustment method such as the size of the inspection interval or the correction threshold $\mathrm{O}_{\mathrm{lim}}$ (see Fig. 5). The parameters used in this article are exemplary to keep the experiment time within reasonable limits and lead to good results for the considered workpiece. Therefore, it is necessary to determine the optimal parameters for general use, independent 
from workpiece properties. A dynamic adjustment of those parameters based on current process parameters, such as the remaining batch size, could further reduce downtimes of the feeding system. Lastly, future work is to be done on the transferability of the described method to other plants and under different environmental conditions.

\section{Summary and outlook}

This contribution introduces a method designed to enable an aerodynamic part feeding system to respond to changing ambient conditions autonomously. This work draws on a genetic algorithm for identifying the optimal configuration of the part feeding system for new workpieces developed and validated in previous research. For the purposes presented here, an altered version of this algorithm was used to adjust the configuration of the feeding system in the case that the initial configuration no longer provides a satisfactory feeding rate. According to extensive studies using a validated simulation model of the feeding system, the performance of the feeding system is influenced by changes in certain ambient conditions (see Fig. 4). To enable the system to counteract these changes autonomously, an adjustment method was developed, based on monitoring the orientation rate in defined intervals and triggering the aforementioned, altered genetic algorithm if necessary (see Fig. 5). This corrective algorithm uses the best individuals from the initial configuration as starting population instead of creating a new, random starting population. This alteration was expected to reduce the setting time of the corrective algorithm compared to the initial algorithm, ensuring efficient operation of the feeding system in changing ambient conditions. The functionality has been demonstrated in this contribution, using offsets for the air pressure and the transfer velocity, as well as fluctuating coefficients of sliding friction as examples of changing ambient conditions. The benefit of the corrective algorithm has been proven in the simulation environment and validated by a test series on the actual aerodynamic feeding system. The continuous review of the orientation rate allows the detection of defined deviations that require a re-parameterisation, which is subsequently processed automatically by triggering the corrective algorithm described above. This algorithm enables the system to adapt autonomously to any changes of ambient conditions-even if the impact factor or its specific influence is not known before. Thus, it may be assumed that the presented approach serves as a basis for developing a resilient part feeding system. To ensure the system's efficiency in practical operation, further research activities must be carried out to determine the optimal values for the length of inspection intervals and the limit of the orientation rate that triggers a re-parameterisation. In addition, current process parameters, like the remaining batch size, should be considered to decide whether a reparameterisation is more efficient than continuing the feeding process with reduced performance.

Acknowledgements The authors would like to thank the German Research Foundation (DFG) for their financial support of the research projects NY 4/51-1, NY 4/51-2 and RA 1736/19-2.

Funding Open Access funding enabled and organized by Projekt DEAL.

Open Access This article is licensed under a Creative Commons Attribution 4.0 International License, which permits use, sharing, adaptation, distribution and reproduction in any medium or format, as long as you give appropriate credit to the original author(s) and the source, provide a link to the Creative Commons licence, and indicate if changes were made. The images or other third party material in this article are included in the article's Creative Commons licence, unless indicated otherwise in a credit line to the material. If material is not included in the article's Creative Commons licence and your intended use is not permitted by statutory regulation or exceeds the permitted use, you will need to obtain permission directly from the copyright holder. To view a copy of this licence, visit http://creativecommons.org/licenses/by/4.0/.

\section{References}

1. Barbazza L, Faccio M, Oscari F, Rosati G (2017) Agility in assembly systems: a comparison model. Assem Autom 37(4):411-421. https://doi.org/10.1108/AA-10-2016-128

2. Boothroyd G (2005) Assembly automation and product design. CRC Press, Boca Raton

3. Busch J (2016) Entwicklung einer intelligenten aerodynamischen Zuführanlage für die Hochleistungsmontage. Tewiss Verlag, Garbsen

4. Busch J, Driehorst M, Nyhuis P (2015) Aerodynamische Orientierung in der Zuführtechnik - mathematische Darstellung und Simulation eines aerodynamischen Orientierungsprozesses. Wt Werkstattstechnik Online 105(1):49-54

5. Busch J, Quirico M, Richter L, Schmidt M, Raatz A, Nyhuis P (2015) A genetic algorithm for a self-learning parameterization of an aerodynamic part feeding system for high-speed assembly. CIRP Ann 64(1):5-8. https://doi.org/10.1016/j.cirp.2015.04.044

6. Edmondson NF, Redford AH (2001) Flexible parts feeding for flexible assembly. Int J Prod Res 39(11):2279-2294. https://doi. org/10.1080/00207540110038487

7. Fantoni G, Santochi M (2010) Development and testing of a brush feeder. CIRP Ann 59(1):17-20. https://doi.org/10.1016/j.cirp. 2010.03.049

8. Fleischer J, Herder S, Leberle U (2011) Automated supply of micro parts based on the micro slide conveying principle. CIRP Ann 60(1):13-16. https://doi.org/10.1016/j.cirp.2011.03.004

9. Frädrich T, Pachow-Frauenhofer J, Torsten F, Nyhuis P (2011) Aerodynamic feeding systems: an example for changeable technology. Assem Autom 31(1):47-52. https://doi.org/10.1108/01445 151111104164

10. Joneja A, Lee NK (1998) Automated configuration of parametric feeding tools for mass customization. Comput Ind Eng 35(34):463-466. https://doi.org/10.1016/S0360-8352(98)00134-X

11. Karl F, Reinhart G (2015) Reconfigurations on manufacturing resources: identification of needs and planning. Prod Eng 9(3):393-404. https://doi.org/10.1007/s11740-015-0607-x 
12. Loy M, Reinhart G (2010) A new modular feeding system and its economic scope of application. Prod Eng 4(4):357-362. https:// doi.org/10.1007/s11740-010-0248-z

13. Man KF, Tang KS, Kwong S (2001) Genetic algorithms: concepts and designs. Springer Science \& Business Media, Berlin

14. Mathiesen S, Sorensen LC, Kraft D, Ellekilde LP (2018) Optimisation of trap design for vibratory bowl feeders. IEEE international conference on robotics and automation (ICRA). IEEE (2018). https://doi.org/10.1109/icra.2018.8460767

15. Reinhart G, Loy M (2010) Design of a modular feeder for optimal operating performance. CIRP J Manuf Sci Technol 3(3):191-195. https://doi.org/10.1016/j.cirpj.2010.09.003

16. Wiendahl HP, Rybarczyk A (2003) Using air streams for part feeding systems-innovative and reliable solutions for orientation and transport. J Mater Process Technol 138(1-3):189-195. https:// doi.org/10.1016/s0924-0136(03)00070-0

17. Zhang T, Smith G, Berretty RP, Overmars M, Goldberg K (2000) The toppling graph: designing pin sequences for part feeding. Proceedings 2000 ICRA. Millennium conference. IEEE international conference on robotics and automation. Symposia proceedings (Cat. No. 00CH37065), vol 1, pp 139-146. IEEE (2000). https:// doi.org/10.1109/ROBOT.2000.844051

Publisher's Note Springer Nature remains neutral with regard to jurisdictional claims in published maps and institutional affiliations. 